\title{
O papel da atenção básica no cuidado ao usuário de crack: opinião de usuários, trabalhadores e gestores do sistema ${ }^{1}$
}

\author{
Leandro Barbosa de Pinho² \\ Débora Schlotefeldt Siniak ${ }^{3}$
}

Objetivou-se conhecer a opinião de usuários do sistema, trabalhadores e gestores sobre o papel da atenção básica no cuidado ao usuário de crack. Recorte de estudo avaliativo, desenvolvido em Viamão-RS, com usuários, familiares, trabalhadores de saúde mental e gestores. Utilizou o referencial teórico e metodológico da Avaliação de Quarta Geração. Foram realizadas entrevistas com a aplicação do Círculo Hermenêutico e Dialético. O método de análise foi o Método Comparativo Constante. Apontou-se a dificuldade de articulação dos serviços especializados com a rede básica, prejudicando acesso e continuidade do cuidado. Destacou-se a escassez de profissionais capacitados para trabalhar com o fenômeno das drogas, limitando o potencial inventivo da rede. Ressalta-se a relevância de investir no matriciamento como mecanismo de integração e educação permanente em saúde mental. Descritores: Atenção Primária à Saúde; Saúde Mental; Cocaína Crack.

\footnotetext{
${ }^{1}$ Apoio financeiro do Conselho Nacional de Desenvolvimento Científico e Tecnológico (CNPq), processo $n^{\circ}$ 402810/2010-4.

${ }^{2} \mathrm{PhD}$, Professor Adjunto, Escola de Enfermagem, Universidade Federal do Rio Grande do Sul, Porto Alegre, RS, Brasil

${ }^{3} \mathrm{MSc}$, Professor Assistente, Universidade Federal do Pampa, Uruguaiana, RS, Brasil.
}

\author{
Correspondência: \\ Leandro Barbosa de Pinho \\ Universidade Federal do Rio Grande do Sul \\ Rua São Manoel, 963 \\ Bairro: Rio Branco \\ CEP: 90620-110, Porto Alegre, RS, Brasil \\ E-mail: Ibpinho@ufrgs.br
}




\title{
The role of primary care in the assistance to crack user: opinion from
} users, collaborators and managers of the system

The objective of this study was to understand the opinion from users of the system, collaborators and managers on the role of primary care in the assistance of crack users. This is part of an evaluative study, developed in Viamão-RS with users, family members, mental health mental health and managers. It was used theoretical and methodological framework of the Fourth Generation Evaluation. Interviews were conducted using the application of the Hermeneutic Dialectic Circle. The Constant Comparative Method was used as analysis. The difficulty of articulation of specialized services with the basic network was pointed out, hindering access and continuity of care. The shortage of trained professionals to work with the drug phenomenon stood out, limiting the inventive potential of the network. The importance of investing in matrix support as integration mechanism and continuing education in mental health is emphasized.

Descriptors: Primary Health Care; Mental Health; Crack Cocaine.

\section{El papel de la atención básica en el cuidado al usuario de crack: opinión} de usuarios, trabajadores y gestores del sistema

\begin{abstract}
Se objetivó conocer la opinión de usuarios del sistema, trabajadores y gestores sobre el papel de la atención básica en el cuidado al usuario de crack. Recorte de estudio evaluativo, desarrollado en Viamão-RS, con usuarios, familiares, trabajadores de salud mental y gestores. Utilizó el referencial teorético y metodológico de la Evaluación de Cuarta Generación. Fueron realizadas entrevistas con la aplicación del Círculo Hermenéutico y Dialéctico. El método de análisis fue el Método Comparativo Constante. Se apuntó la dificultad de articulación de los servicios especializados con la red básica, perjudicando acceso y continuidad del cuidado. Se destacó la escasez de profesionales capacitados para trabajar con el fenómeno de las drogas, limitando el potencial inventivo de la red. Se resalta la relevancia de invertir en el apoyo matricial como mecanismo de integración y educación permanente en salud mental.
\end{abstract}

Descriptores: Atención Primaria de Salud; Salud Mental; Cocaína Crack.

\section{Introdução}

Dentro das premissas que sustentam a reforma psiquiátrica, no referente ao paradigma de cuidado no campo psicossocial, é possível entender a importância da compreensão de que a loucura não pode ser reduzida a apenas um único olhar, considerando-se que possui influência multifatorial. Nesse sentido, diferente das verdades constituídas ao longo da era da psiquiatria tradicional, atualmente vem-se trabalhando para a ampliação dos dispositivos de cuidado que garantam qualidade e valorização do sujeito e suas diferenças ${ }^{(1)}$.

Se considerarmos os atuais serviços da rede de saúde mental como aqueles que procuram romper com o circuito manicomial, responsável pelas iatrogenias e com caráter antiterapêutico, a análise da acessibilidade se configura considerandose instrumento para compreender a qualidade e o potencial do serviço de produzir transformações na realidade do cuidado(2). 
Neste diálogo tão importante, o uso de drogas não se desvincula considerando-se necessidade concreta de repensar o campo da saúde. Sabe-se que qualquer intervenção nesta área se baseia, em sua maioria, na certeza dos danos provocados pelo seu consumo, sendo essa visão quase que universal. Entre a exclusão do usuário de drogas ou a adoção da modalidade da abstemia como única alternativa de tratamento, o melhor a fazer seria entender a relação com a droga ou sua demanda, em uma tentativa de resgatar o poder do sujeito nessa relação. É como se o mistério na resolução dos problemas com o uso da droga estivesse na palidez existente entre as políticas assépticas, padronizadoras e a desconsideração de estilos individuais e singulares ${ }^{(3)}$.

Nesse contexto, residem também as tendências mais atuais (e não menos contraditórias) em relação ao cuidado ao usuário de crack. Sabemos da magnitude das repercussões causadas pelo uso da substância na vida do usuário $^{(4)}$, no cotidiano das famílias ${ }^{(5)}$ e na constituição de políticas públicas mais sensíveis e específicas à demanda(6). No entanto, entendemos que, em uma definição de cuidado baseado na integralidade, é preciso investir nas distintas possibilidades territoriais, pois o fenômeno do uso de drogas faz parte de nossa sociedade.

Portanto, ressaltamos a evidência de uma rede de atenção psicossocial como estratégia capaz de proporcionar acesso aos cuidados para indivíduos com problemas decorrentes do consumo da droga. Uma rede não apenas formada por Centros de Atenção Psicossocial (CAPS), mas também pela rede básica, pois, quando articulada ao serviço especializado, pode fortalecer $\mathrm{o}$ cuidado em liberdade ${ }^{(7)}$. $E$, nessa composição, entendemos que desponta o matriciamento como dispositivo que possa garantir essa conexão, de modo a favorecer a continuidade do cuidado fora dos muros tradicionais dos serviços.

Diante do exposto, este estudo tem por objetivo analisar a opinião de usuários do sistema, trabalhadores e gestores sobre o papel da atenção básica no cuidado ao usuário de crack.

\section{Método}

Trata-se de um recorte da pesquisa "Avaliação qualitativa da rede de serviços em saúde mental para atendimento a usuários de crack (ViaREDE)", financiada pelo CNPq/Ministério da Saúde. Possui natureza avaliativa, do tipo estudo de caso e foi desenvolvida no município de Viamão-RS. Baseou-se na utilização da Avaliação de Quarta Geração( ${ }^{(8)}$ como referencial teórico e metodológico.

A Avaliação de Quarta Geração propõe uma avaliação construtivista responsiva. O termo responsivo é utilizado para designar um caminho diferente de focalizar a avaliação, delimitado por meio de um processo interativo e de negociação que envolve grupos de interesse. O termo construtivista, também chamado de interpretativo ou hermenêutico, é um modo responsivo de focar e um modo construtivista de fazer ${ }^{(8)}$.

Os sujeitos do estudo foram os oito trabalhadores do Centro de Atenção Psicossocial - Álcool e outras Drogas (CAPS AD), dez usuários do serviço, onze familiares de usuários e sete gestores do sistema. Os critérios de inclusão dos profissionais e dos gestores foram o fato de trabalharem no CAPS AD e na gestão de saúde mental do município há pelo menos seis meses, respectivamente. Os critérios de inclusão dos usuários foram: frequentarem o CAPS AD ou de neste momento terem frequentado outro serviço da rede de saúde mental em função do uso de crack; estarem com boas condições de comunicação e que se dispusessem voluntariamente a participar da pesquisa, além de não estar sob condições clínicas que prejudicassem sua entrevista. Quanto aos familiares, os critérios consistiram em acompanhar ou ter acompanhado algum parente, usuário de crack, em atendimento no CAPS AD e em outro ponto da rede de serviços de saúde mental.

Em relação aos critérios de exclusão, os trabalhadores e gestores não poderiam estar gozando de licença ou férias no período da coleta. No referente aos usuários e familiares, foram excluídos os que estivessem em estado psicótico ou sem condições cognitivas para participar da entrevista.

Os integrantes da equipe foram identificados com a inicial "E", os gestores com a inicial "G", os usuários com a inicial "U" e os familiares com a inicial " $F$ ", sendo as iniciais seguidas pela ordem em que apareceram na entrevista, como por exemplo: E3, F4, U2, G5.

A coleta de dados ocorreu de janeiro a março de 2013, através de observações de campo e entrevistas. As observações de campo totalizaram 189 horas, sendo registradas em um diário de campo. Com relação às entrevistas, ao todo foram realizadas 36 .

As entrevistas foram realizadas com a aplicação do Círculo Hermenêutico Dialético. Esse dispositivo é chamado de hermenêutico porque é interpretativo e de dialético porque representa a comparação e o contraste das visões, para a realização de um alto nível de síntese ${ }^{(8)}$. O método utilizado exigiu que a análise e a coleta de dados fossem processos paralelos, um direcionando o outro, baseado no Método Comparativo Constante ${ }^{(9)}$

Depois da coleta de dados e a organização das construções de cada grupo, realizou-se a etapa da negociação. Foram reunidos os entrevistados, sendo apresentado o resultado provisório da análise dos 
dados, para que pudessem ter acesso à totalidade das informações e tivessem a oportunidade de modificálas ou de afirmar a sua credibilidade( ${ }^{(8)}$.

A partir da negociação, os pesquisadores procederam à etapa final de análise dos dados. Nela, as questões surgidas foram reagrupadas, permitindo a construção de categorias temáticas. Os resultados deste estudo foram organizados a partir da temática "acesso à rede", no qual convergiram questões relacionadas ao papel da atenção básica e do matriciamento na composição de redes de atenção psicossocial para o cuidado ao usuário de crack.

O projeto foi submetido à avaliação pelo Comitê de Ética em Pesquisa da Universidade Federal do Rio Grande do Sul (UFRGS), recebendo parecer favorável à execução (protocolo 20157/2011). Foi também, por solicitação do CEP/UFRGS, avaliado pelo CONEP/ MS, recebendo parecer favorável à sua execução (parecer 337/2012).

\section{Resultados}

A atenção básica foi avaliada pelos grupos de interesse como um dos pontos frágeis da rede em relação ao cuidado ao usuário de crack, principalmente pela dificuldade de encaminhamento aos serviços especializados e à falta de profissionais capacitados para o entendimento e para a intervenção sobre o fenômeno das drogas, como exemplificado nas falas a seguir:

[...] Isso, sim, porque se tivesse nos postos [profissionais capacitados para atender dependentes químicos], de lá tu tem como encaminhar pro CAPS. [...] Nos postos, até de repente uma emergência, a gente vai passar na frente de alguém que estivesse mais necessitado, mas se estivesse nos postos ajudaria bastante (U2)

[...] Lá não tem isso (profissionais que entendem de drogas). Acho que seria melhor se tivesse lá, melhor que vir direto pro CAPS. (U3)

Familiares também apontam que a atenção básica no município é um dos recursos com os quais menos podem contar quando precisam. Em geral, ressaltam que essa questão aparece mais quando envolve o uso dos serviços de saúde em situação de urgência. Segundo eles, os postos de saúde não funcionam, e, quando isso acontece, o funcionamento é típico dos modelos tradicionais "por fichas", como apontam os discursos:

[...] o posto de saúde que é perto da minha casa a gente tem que dormir lá para poder conseguir uma ficha, que é terrível porque não dá nem para ficar na fila por causa dos assaltos, e lá dentro no momento que a gente consegue uma ficha até que é bem atendido, mas até tu conseguir chegar lá tem que passar muito trabalho antes e se tiver ficha pra ti consultar. (F2)

[...] Posto de saúde não funciona de fato, não funciona. (F10)

Aideia de mudança de modelo, atrelada à Atenção Básica, mais especificamente à Estratégia Saúde da
Família, ainda parece um desafio a Viamão, que possui algumas Unidades de Saúde da Família operando na lógica de modelos tradicionais de Unidades Básicas de Saúde (UBS). Perde-se um importante mecanismo de acesso do usuário ao sistema de saúde mental, uma vez que o usuário de crack possui uma rotina de funcionamento diferente das unidades de saúde territoriais:

[...] E aí o usuário de crack, do modo como eles acionam o tempo, nunca vai usar, porque ele tem que ir às $3 h$ da manhã e esta hora ele está indo para a boca, não está indo para o posto pegar ficha e pela manhã ele dorme, talvez procure o posto às $4 h$ da tarde. (E7)

Outra questão importante que os grupos de interesse avaliam é a necessidade de investir na educação permanente dos trabalhadores, para compreenderem o funcionamento do usuário e suas necessidades. É o que U9 aponta em seu depoimento a seguir:

[...] tinha que ter um CAPS em cada vila, porque as vilas são horríveis. Onde eu morava tem um ponto de droga 24h, ai é fumódromo $24 \mathrm{~h}$ porque não tem alguém para apoiar esse pessoal. Muitos querem ser ajudados, muitos não querem, infelizmente acontece. [...] Ou nos postos alguém que faz esse tipo de trabalho, nos postos de saúde, seria melhor [...] Colocar nos postos de saúde que já tenha trabalhado com esse tipo de pessoa, com dependência, para quando chegar no posto, poder conversar e instruir as pessoas, muitas vezes cheguei no posto e disse para a pessoa que era dependente químico e deu preconceito, eu senti o preconceito daquela pessoa naquele momento. [...] Gente preparada nos postos de saúde pra tratar esse tipo de pessoas, isso sim. (U9)

Os grupos de interesse avaliam que é necessário investir nessas ações de educação permanente, no sentido de diminuir preconceitos, estigmas, reorientar modelos e atuar nas situações de despreparo técnico do profissional. Para os trabalhadores do CAPS ad, a maior dificuldade com as equipes da atenção básica está nesse tratamento nos cenários de vida do usuário, uma vez que o profissional da rede se "assusta" com a realidade do crack e encaminha direto aos serviços especializados, como vemos nestes depoimentos:

[...] Quando vem pela UBS acho que eles lá se assustam muito, é da equipe direto o encaminhamento tem alguma questão de neste sentido é encaminhamento... É bem difícil, é bate volta né, quando bate lá. Muitas vezes a gente da alta, encaminha pra UBS, o usuário acaba recaindo pra acessar o serviço de novo porque ele sente acolhido aqui e não lá... (E3)

O entrevistado G3, por exemplo, avalia que são desenvolvidas atividades de educação permanente na rede para diminuir a distorção desses encaminhamentos ou recusas, embora pense que elas precisam ser potencializadas e tornadas mais visíveis na realidade do município, como revela:

[...] A gente já está fazendo que é a educação permanente nesse serviços pra gente poder entender todo esse movimento que existe na Saúde Mental dessa linha de cuidado da Saúde Mental 
do país tá imprimindo. [...] Eu acho que a educação permanente ela vem pra gente poder entender melhor, se informar melhor em relação a isso, o preconceito ao uso dessa substância específica... (G3)

Contribuindo para minimizar essa problemática, destacamos o matriciamento considerando-se dispositivo de composição dessas conexões existentes entre o serviço especializado e a rede básica de saúde. No entanto, em Viamão, o matriciamento ainda possui uma organização centrada em protocolos tradicionais de cuidado. O único profissional que fazia parte da equipe, no momento da coleta de dados, era um médico psiquiatra, que dava assessoria a unidades básicas de saúde, prestando atendimento, quando a lógica seria de corresponsabilização.

Uma das questões que apareceu no decorrer da aplicação do círculo foi a de que o matriciamento ainda precisa se estruturar considerando-se estratégia de cuidado na rede. As pequenas ações ainda estariam voltadas para os transtornos psiquiátricos mais comuns, não havendo, segundo os depoimentos dos trabalhadores, uma longitudinalidade em relação aos problemas com álcool e outras drogas:

[...] Outra ferramenta [matriciamento] importante, mas que nunca conseguiu se estruturar, ou porque as pessoas não queriam fazer parte né?... (E3)

[...] Assim a gente sabe que tem o matriciamento que é o doutor que faz, o psiquiatra ali do CAPS II, mas eu acho que nada voltado para $A D$ [Álcool e Drogas]... (E4)

[...] Eu acho que talvez seja um dos mais caros e profundos problema aqui, o matriciamento. Nós temos hoje na Saúde Mental um matriciador, o doutor [...], e é ele que faz o matriciamento da Saúde Mental. Não que eu acho que o matriciamento se faz só com uma equipe de matriciamento, nosso matriciamento é todo dia. Mas eu acho que o matriciamento precisa de uma equipe que possa fazer esse trabalho com as unidades e com a rede. Hoje em Viamão é muito incipiente, muito pequeno o trabalho que se faz e muitas vezes equivocado porque o que acontece daqui a pouco o matriciador vai pras unidades fazer atendimentos e não matriciamentos. [...]. (G3)

Não existe matriciamento em Viamão. [...] o matriciamento pra nós é básico e é contextual. O nosso matriciamento [CAPSi] não é igual ao deles [CAPS adulto], é outro, a gente tem que ver no bairro, o que a gente tem, as escolas, apesar da gente centralizar, todo o Viamão vem pra cá. Porque esse matriciamento, vocês sabem né, tem que ter uma comissão, eles já vieram aqui umas duzentas vezes e nunca funcionou. Matriciamento é tu falar, fazer uma capacitação com o pessoal do posto. Eu acho que teria que ter uma comissão, bem objetiva do que a gente precisa: que lugar que nós estamos? Como que funciona Viamão? Como é que a colonização? Qual a necessidade desse matriciamento? Quem é aquele pessoal do posto? O matriciamento tem que ser contextualizado e exige constantemente, uma equipe especializada. Não pode pegar 12 lá e, não a gente tem que ter uma equipe de matriciamento fora, sabe um matriciamento, um departamento, uma coisa tipo PSF [Programa Saúde da Família] ou até fazer esse matriciamento com PSF. (G4)

Apesar de raras e pontuais ações em saúde, sendo consenso nos grupos de interesse dos trabalhadores e dos gestores a necessidade de fortalecimento, o matriciamento é compreendido como um disparador da rede e um dispositivo de integração, devendo ser cuidadosamente planejado e repensado na política municipal de saúde:

[...] Acho que é muito importante o matriciamento e isso também eu não vejo isso em primeiro plano na secretaria. O interesse deles mesmo é fortalecer a atenção básica e eu vejo que só fortalecer a atenção básica não é o suficiente, tem todo um contexto, porque a atenção básica tá ligada com tudo. (E6)

[...] Ele visita então as Unidades ou as Estratégias da Família pra fazer essa discussão com as equipes das unidades básicas e com os usuários. Eu diria assim, hoje no município o matriciamento, ele ainda não está concretizado da forma que deveria se dar o matriciamento. A gente tem pensado muito como colegiado, como coordenadores, a gente envolver mais as equipes dos CAPS como um todo pra que isso possa se dar como uma proposta do CAPS, não somente nas quintas-feiras a tarde, mas que isso possa se dar a qualquer momento que há necessidade de se trabalhar com a proposta de matriciamento. Então eu ainda acho que teria que se dar outro olhar pro matriciamento. Se dá muito pouco, acontece muito pouco, mas eu acho que pode, acredito que ainda tem que melhorar bastante. No momento que a gente conseguir ir a cada quinze dias numa unidade básica, com uma proposta de grupo, de espaço assim, de educação em saúde mental, prevenção, promoção, acho que isso é muito legal. (G2)

\section{Discussão}

A qualidade do cuidado está relacionada diretamente à capacidade de acolhimento e satisfação do usuário. Não basta apenas realizar procedimentos técnicos, perguntar sobre as queixas e orientar. $\mathrm{O}$ usuário deseja ser compreendido em seu problema, deseja uma solução. Nesse sentido, o trabalhador deve-se deixar afetar pelos problemas do usuário, flexibilizando a clínica e proporcionando a real mudança de modelo(10-11).

A educação permanente dos trabalhadores atua como uma estratégia pedagógica para lidar com os problemas de saúde dos indivíduos e dos coletivos. Em vários estudos ${ }^{(12-14)}$ no âmbito da atenção básica $e$ da saúde mental essa questão aparece também como uma tecnologia do processo de trabalho em saúde, capaz de provocar tensionamentos e transformações na realidade assistencial.

Entendemos que há distintas possibilidades de oferta de educação permanente aos trabalhadores da rede de saúde de Viamão. Considerando que a atenção básica é fundamental nessa tessitura de rede, visualizamos que o matriciamento pode ser incorporado ao cotidiano da atenção básica para dar seguimento e respaldo à atuação do trabalhador, no sentido de fazer "matriciamentos" e não "atendimentos", como ressaltava o entrevistado 
G3. Sendo reconstituído com outras metodologias de trabalho, como discussão de casos compartilhados e composição de mini-equipes de suporte do CAPS à rede, o próprio matriciamento fortalecerá a atenção básica e permitirá que o usuário não se desloque aos serviços especializados, salvo em casos essenciais. Assim, consideramos que se promoverá a costura da rede, facilitando o trânsito do usuário por ela.

O matriciamento em saúde é, por natureza, uma estratégia que tem por objetivo assegurar retaguarda especializada a profissionais e equipes responsáveis pela atenção no campo da saúde. Define-se como uma metodologia de trabalho complementar àquela existente em sistemas hierarquizados, que utilizam mecanismos de referência e contra-referência, centros de regulação e protocolos. $\mathrm{O}$ apoio matricial objetiva oferecer suporte técnico pedagógico e retaguarda assistencial especializada às equipes de referência, dependendo da construção compartilhada de diretrizes sanitárias e clínicas ${ }^{(15)}$.

Em uma proposta de rede, o apoio matricial surge como dispositivo que permite a interlocução entre os equipamentos de assistência à saúde e os demais serviços internos e externos à rede ${ }^{(16)}$. Trata-se de uma estratégia que otimiza o processo de trabalho, organiza-o no conjunto dos serviços e procura promover uma horizontalidade nas relações de trabalho, corresponsabilizando os atores na construção dos projetos terapêuticos.

O profissional que atua na prática do matriciamento deve estar preparado para trabalhar com grupos heterogêneos e com possibilidades que vão além de ações centradas no campo da saúde. Deve ser aberto às novas descobertas que residem no compartilhamento de poderes e saberes, considerando a complexidade das experiências dos usuários no campo do adoecimento e criando, com isso, ações multidisciplinares de prevenção e promoção da saúde ${ }^{(17)}$.

Os trabalhadores e gestores possuem a compreensão de que o matriciamento pode ajudar na integração de distintos pontos da rede, em especial a atenção básica, ainda frágil no município. Complementada pela avaliação de usuários e familiares, que sinalizam as restrições de acesso à rede básica, consideramos que é preciso repensar essa articulação no contexto locorregional.

No campo de álcool e outras drogas, mais ainda o matriciamento precisa se constituir nesse escopo. Ele deve ser entendido como estratégia de processo de trabalho, não apenas reduzido ao trabalho de um membro da equipe. Matriciamento se faz no dia a dia, em um telefonema, em uma orientação pedagógica com as várias facetas da rede, como uma estratégia de educação permanente em saúde mental. Só assim percebemos que seja possível realmente valorizar o uso de drogas como fenômeno multifatorial e que, via de regra, prescinde de um cuidado integrado e atrelado às premissas da atenção psicossocial.

\section{Conclusão}

Sabe-se que a atenção básica configura-se muitas vezes como porta de entrada para o usuário da saúde mental em sua trajetória na busca pela resolução de problemas. No entanto, as fragilidades nessa porta de acesso muitas vezes traduzem-se em intervenções focadas no serviço especializado, desvinculando a ideia ampla do caráter de cuidado em rede.

Os resultados deste estudo apontaram para a necessidade de capacitação dos profissionais de saúde que atuam na rede básica, promovendo interlocução intersetorial através de ações de matriciamento e também de educação permanente, como tecnologias que possam redimensionar os processos de trabalho e cuidar dentro das premissas do campo psicossocial.

O desenvolvimento de ações compartilhadas, voltadas para a interação da equipe e a criação de espaços estratégicos para que ocorra essa interação, despontam como uma necessidade dentro do município. Desta forma, pensamos que é preciso ampliar essas ações que possam evidenciar o sentimento de corresponsabilização, no sentido não apenas de considerar a complexidade do fenômeno do uso do crack no mundo contemporâneo, mas também a necessidade de repensar o processo de trabalho em saúde mental, enfrentando a fragmentação.

Parece claro que a dificuldade de articulação em rede é uma preocupação candente para o grupo de gestores e trabalhadores, o que não deixa de ser positivo. A reflexão da necessidade dessa parceria acaba trazendo novas possibilidades para o município, mostrando que o debate técnico e político deles é comprometido e articulado com as atuais tendências de cuidado no campo psicossocial.

Em relação aos limites do estudo, entendemos que precisamos avançar mais na possibilidade de fazer intervenções na realidade, em parceria com os grupos de interesse, como preconiza a Avaliação de Quarta Geração. A despeito da importância do plano descritivo, como apresentado aqui, é preciso construir melhor uma agenda de ideias e fundamentos comprometidos com mudanças sociais nas políticas de saúde mental. Só assim fecharemos um ciclo em que a avaliação participativa trará substantivas contribuições para o cenário, às relações e aos sujeitos.

Esperamos este estudo possa dar uma dimensão mais precisa sobre as diferentes facetas que envolvem o uso do crack, mais especificamente sobre as repercussões do fenômeno nos processos de trabalho em saúde mental e nas políticas públicas locais. 


\section{Referências}

1. Amarante P. Saúde mental e atenção psicossocial. 4th ed. Rio de Janeiro: Fiocruz; 2013. 123 p.

2. Pinho LB, Kantorski LP, Bañon-Hernández AM. O discurso sobre o acolhimento e a acessibilidade nos serviços comunitários de saúde mental. Cogitare Enferm. 2009;14(4):612-9.

3. Alarcon S. A síndrome de Elêusis: considerações sobre as políticas públicas no campo da atenção ao usuário de álcool e outras drogas. In: Alarcon $\mathrm{S}$, Jorge MAS, editors. Álcool e outras drogas: diálogos sobre um mal-estar contemporâneo. Rio de Janeiro (RJ): Fiocruz; 2012. p. 45-62.

4. Oliveira LG, Nappo SA. Crack na cidade de São Paulo: acessibilidade, estratégias de mercado e formas de uso. Rev Psiquiatr Clin. 2008;35(6):212-8.

5. Borba LO, Schwartz E, Kantorski LP. A sobrecarga da família que convive com a realidade do transtorno mental. Acta Paul Enferm. 2008;21(4):588-94.

6. Ministério da Saúde (BR). Decreto $n^{0} 7.179$, de 20 de maio de 2010: Institui o Plano Integrado de Enfrentamento ao Crack e outras Drogas, cria seu Comitê Gestor, e dá outras providências. Brasília (DF): Ministério da Saúde; 2010.

7. Soares $M H$. A inserção do enfermeiro psiquiátrico na equipe de apoio matricial em saúde mental. SMAD, Rev Eletr Saúde Mental Alcool Drog. [Internet]. 2008 [acesso 12 dez 2015];4(2):1-12.Disponível em: http://pepsic. bvsalud.org/scielo.php?script=sci_arttext\&pid=S180669762008000200006\&lng=pt\&tlng=pt.

8. Guba EG, Lincoln YS. Avaliação de Quarta Geração. 1st ed. Campinas (SP): UNICAMP; 2011. 320 p.

9. Kolb SM. Grounded Theory and the constant comparative method: valid research strategies for educators. J Emerging Trends Educ Res Policy Studies (JETERAPS). 2012;3(1):83-6.

10. Medeiros FA, Araújo-Souza GC, AlbuquerqueBarbosa AA, Clara-Costa IC. Acolhimento em uma Unidade Básica de Saúde: a satisfação do usuário em foco. Rev Saúde Pública. 2010; 12(3):402-13.

11. Matumoto S, Fortuna CM, Kawada LS, Mishima SM, Pereira MJB. Mapping pain in the clinical practice of nurses within primary. Texto Contexto Enferm. 2013;22(2):318-26.

12. Meirelles MCP. Educação permanente em saúde no processo de trabalho de um Centro de Atenção Psicossocial [dissertation]. Pelotas (RS): Universidade Federal de Pelotas; 2009. 128 p.

13. Novaes MA, Machiavelli JL, Verde FCV, Campos AS Filho, Rodrigues TRC. Tele-educação para educação continuada das equipes de saúde da família em saúde mental: a experiência de Pernambuco, Brasil. Interface (Botucatu). 2012;16(43):1095-106.

14. Silva ER, Castilho JCN, Fassheber VB. A construção de um projeto coletivo de educação continuada em saúde mental: a experiência do CERSAM-LESTE. Cad Bras Saúde Mental. 2012;4(10):174-6.

15. Ministério da Saúde (BR). Guia prático de Matriciamento em Saúde Mental. Brasília (DF): Ministério da Saúde; 2011.

16. Gomes VG. Apoio matricial: estratégia de interlocução na rede de saúde de Campinas/SP [monografia]. Campinas (SP): Universidade Estadual de Campinas; 2006. 13 p.

17. Barban EG, Oliveira AA. O modelo de assistência da equipe matricial de saúde mental no programa saúde da família do município de São José do Rio Preto (Capacitação e educação permanente aos profissionais de saúde na atenção básica). Arq Ciênc Saúde UNIPAR. 2007;14(1):52-63. 